\section{A) Check for updates}

Cite this: Org. Chem. Front., 2021, 8 4399

Received 27th April 2021, Accepted 12th June 2021

DOI: $10.1039 /$ d1qo00643f

rsc.li/frontiers-organic

\title{
Aqueous assembly of a (pseudo)rotaxane with a donor- $\pi$-acceptor axis formed by a Knoevenagel condensation $\dagger$
}

\author{
Laurent Jucker, ${ }^{a}$ Yves Aeschi $^{\mathrm{a}}$ and Marcel Mayor (D) *a,b,c \\ The incorporation of a linear $D-\pi-A$ "push-pull" chromophore synthesized by a Knoevenagel conden- \\ sation as axle of a rotaxane is reported. While the introduction of the mechanical stoppers by the revers- \\ ible and thermodynamically controlled Knoevenagel reaction turned out to be challenging, mechanical \\ fixation of the superstructure is achieved by "click" chemistry in aqueous solution. The isolated rotaxane \\ with a red- to NIR-emitting dye as axle displays enhanced emission in aqueous buffered media compared \\ to the unthreaded model chromophore.
}

\section{Introduction}

The influence of macrocyclic hosts on the optical properties of their guests (and vice versa) has been investigated in a wide range of supramolecular systems with various emerging applications. Depending on the particular implementation, fluorescence enhancement, electron and/or energy transfer mechanisms can be incorporated in optical triggered molecular switches, typically as a function of the proximity of the components involved. ${ }^{1-4}$ Commonly employed dyes include rylene diimides, ${ }^{5-7}$ naphthalene imides, ${ }^{8}$ azo dyes,${ }^{9-11}$ squaraines, ${ }^{12,13}$ and poly-aromatic hydrocarbons like anthracene ${ }^{14,15}$ or pyrene. ${ }^{16}$ Despite the growing library of rotaxanes, only very few examples comprising linear donor- $\pi-$ acceptor "push-pull" chromophores are reported. ${ }^{17-23}$ An even more surprising fact considering their synthetic accessibility and tunability of their optical properties. ${ }^{24}$

The decoration of a pseudorotaxane with a bulky substituent as stopper to mechanically fix the macrocyclic host is a common assembly strategy for rotaxanes, often referred to as "capping" or "stoppering". A variety of coupling reactions have been employed, with the copper(I)-catalysed azide-alkyne 1,3cycloaddtion (CuAAC) as "click" reaction being particularly popular, ${ }^{10,16}$ combining mild conditions and the concomitant

\footnotetext{
${ }^{a}$ Department of Chemistry, University of Basel, St. Johanns-Ring 19, 4056 Basel, Switzerland. E-mail: marcel.mayor@unibas.ch

${ }^{b}$ Institute for Nanotechnology (INT), Karlsruhe Institute of Technology (KIT), P. O. Box 3640, 76021 Karlsruhe, Germany

${ }^{c}$ Lehn Institute of Functional Materials (LIFM), School of Chemistry,

Sun Yat-Sen University (SYSU), Guangzhou 510275, China

$\dagger$ Electronic supplementary information (ESI) available. See DOI: 10.1039/ d1qo00643f
}

tolerance for a large variety of functional groups with high yields, especially in (metal-)templated assembly strategies. ${ }^{25-28}$ However, in contrast to the irreversible CuAAC, only stoppering by reversible transformations might provide thermodynamic control over the formed superstructures. So far mainly imine condensations ${ }^{29,30}$ and olefin metatheses ${ }^{31}$ have been used for this purpose. To the best of our knowledge, examples taking advantage of the dynamic nature of the Knoevenagel condensation $^{32}$ are limited to the syntheses of two rotaxanes ${ }^{22,23} \mathrm{com}$ prising cyclodextrin-encapsulated cyanine dyes under aqueous basic conditions. More frequent use of the Knoevenagel condensation is observed in the field of covalent organic frameworks ${ }^{33,34}$ and macrocyclizations. ${ }^{35}$

We thus explored the potential of the Knoevenagel condensation as stoppering reaction for pseudorotaxanes assembled in water (Scheme 1a). We not only hoped for the reversible, thermodynamically controlled assembly of rotaxanes, but also wanted to profit from the strong electron accepting nature of the formed olefin decorated with a pair of electron-withdrawing groups (EWG). Our focus was set on the formation of linear donor- $\pi$-acceptor $(D-\pi-A)$ "push-pull" chromophores as axles with emission in the red to near-infrared region (NIR). This emission range is not only of interest for bioimaging, ${ }^{13,36}$ but also particularly appealing for spectroscopy experiments enhanced by gold nanostructures. ${ }^{37,38}$

As macrocycle forming (pseudo)rotaxanes with these axes, the Diederich-type cyclophane $2^{39-41}$ combining strong complexation of hydrophobic guests with water-solubility was considered. Previously, 2 has been employed in rotaxanes comprising oligo(phenylene-ethynylene) (OPE) molecular wires, ${ }^{39,42,43}$ azo dyes, ${ }^{9}$ or naphthalenes ${ }^{44}$ as guests. We have previously used an OPE-functionalized modification of 2 to assemble 
a)

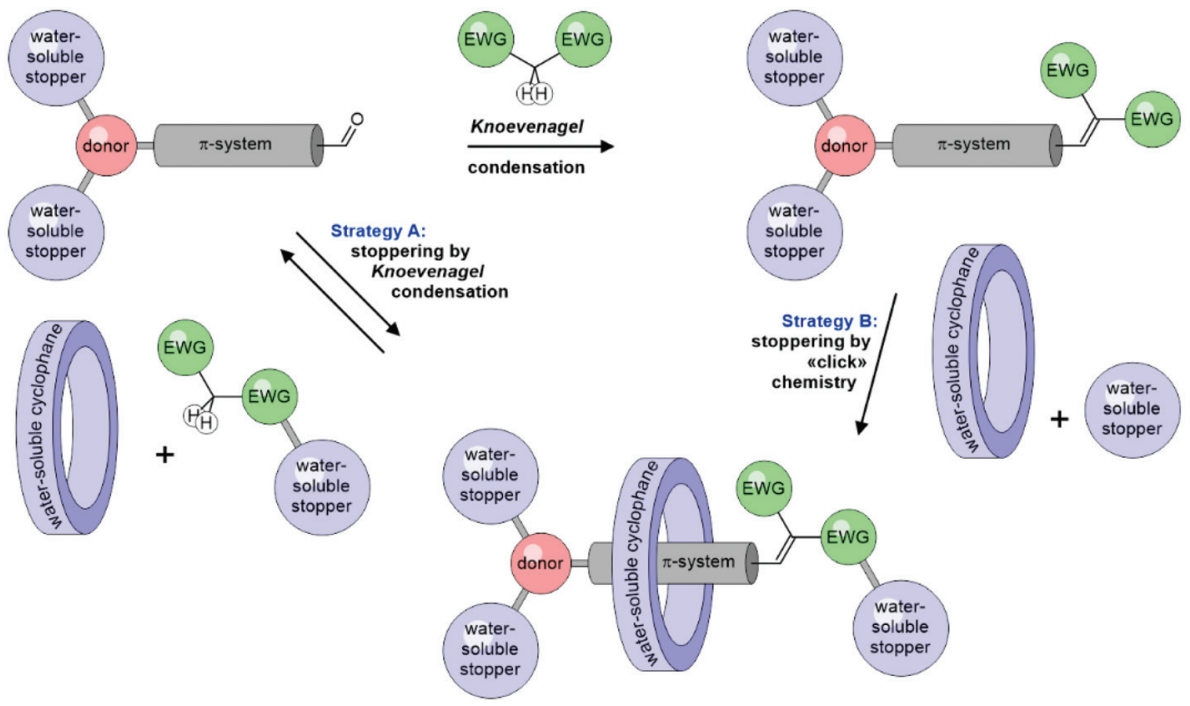

b)

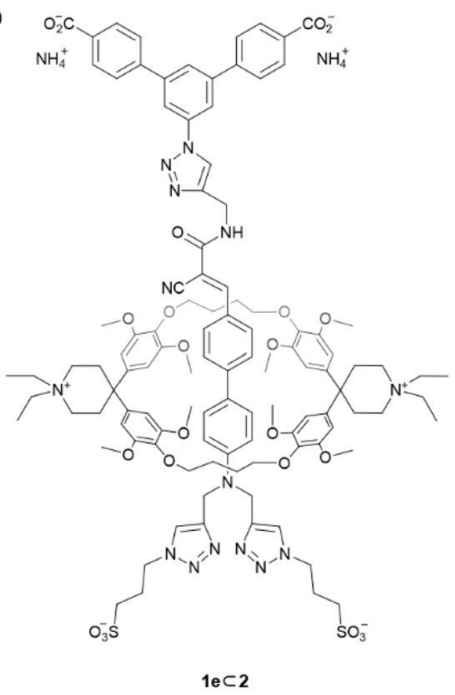

Scheme 1 (a) Both strategies to assemble rotaxanes with a $D-\pi-A$ chromophore as axis considered. (b) Rotaxane 1 ec 2 obtained via strategy $B$.

dimeric, zwitterionic daisy chains in water ${ }^{45}$ and studied the slow association of 2 with carboxylate-terminated OPE structures forming kinetically inert pseudorotaxanes. ${ }^{46}$ Interestingly, the changes observed in the emission intensity upon complexation with 2 strongly depend on the nature of the guest and seem not to be fully understood yet. This further motivated the here reported approach, as axles assembled via Knoevenagel condensation might provide access to a series of (pseudo)rotaxanes incorporating "push-pull” $\mathrm{D}-\pi-\mathrm{A}$ systems of various acceptor strengths.

Here we report our attempts to assemble water-soluble linear "push-pull" chromophores by Knoevenagel condensations as potential axes of rotaxanes. We present our initial struggles to balance reactivity, stability and solubility in water together with the first "proof-of-existence" rotaxane $\mathbf{1 e \subset 2}$ (Scheme 1b) with the desired axis structure. While this mechanically interlinked model compound enabled preliminary investigations of the physical properties of this class of rotaxanes, it was obtained applying a stepwise strategy, assembling first the axis with a Knoevenagel condensation and subsequently fixing the supermolecule by CuAAC "click" chemistry (strategy B in Scheme 1a).

\section{Results and discussion}

\section{Precursor synthesis and pseudorotaxane formation}

With the intention to profit from the Knoevenagel condensation as a stoppering reaction, the aldehyde decorated precursor 3 combining multiple key components was designed (Scheme 2). Its biphenyl backbone acts as both, $\pi$-system of the chromophore bridging donor and acceptor substituent and as hydrophobic station complexing the cyclophane. The electron-donating nitrogen is functionalized with two triazol units bearing sulfonates, which have a double role too, serving as mechanical stopper and as water-solubilizing groups at the same time.

Starting from 4-iodoaniline 4 , two-fold alkylation with propargyl bromide in acetonitrile gave 5 in $66 \%$ yield. Formation of 3-azidopropane-1-sulfonate by $\mathrm{S}_{\mathrm{N}} 2$ of 1,3-propanesultone with sodium azide and subsequent CuAAC gave the watersoluble 6 as the disodium salt in 45\% yield after decomposition of excess azide, purification by reverse-phase column chromatography, and precipitation from water with EtOH. After unsuccessful attempts in commonly employed mixed aqueous-organic media, we found suitable conditions ${ }^{47}$ for the Suzuki-Miyaura cross-coupling of 6 with 4-formylphenylboronic acid in water in absence of a ligand to give the desired aldehyde 3 as the ammonium salt in 78\% yield after reversephase column chromatography.

The interaction between the one-sidedly stoppered watersoluble biphenyl 3 and the cyclophane 2 was investigated by ${ }^{1} \mathrm{H}-\mathrm{NMR}$ spectroscopy (Scheme S1 $\dagger$ ). Addition of an equimolar amount of 2 to a solution of 3 in $\mathrm{D}_{2} \mathrm{O}$ displayed the typical upfield shift of the protons encircled by the host expected for the formation of $3 \subset 2$, along with a broadening of the signals. The aryl signals of the biphenyl system are shifted up-field by 0.5-2.2 ppm, with the central two protons experiencing the strongest shielding effect (as determined by NOESY, Fig. S1 $\dagger$ ). The aldehyde singlet only shifts up-field by $0.36 \mathrm{ppm}$ and the triazole and propyl signals of the stopper are slightly deshielded. According to the extent of the experienced shielding we hypothesized the macrocyclic host in $3 \subset 2$ to be mainly located around the centre of the biphenyl subunit, with the terminal aldehyde accessible for further reactions. A dilution series of $3 \subset 2$ in $\mathrm{D}_{2} \mathrm{O}$ (Fig. $\mathrm{S} 2 \dagger$ ) in the range of $1 \mathrm{mM}$ to $0.1 \mathrm{mM}$ only showed a slight shift of the signals, indicating a high association constant $\left(K_{\mathrm{a}}\right)$ in aqueous solution $\left(K_{\mathrm{a}}>10^{5} \mathrm{M}^{-1}\right){ }^{48}$ This is in line with previously published pseudorotaxanes comprising $2 .^{46}$ 

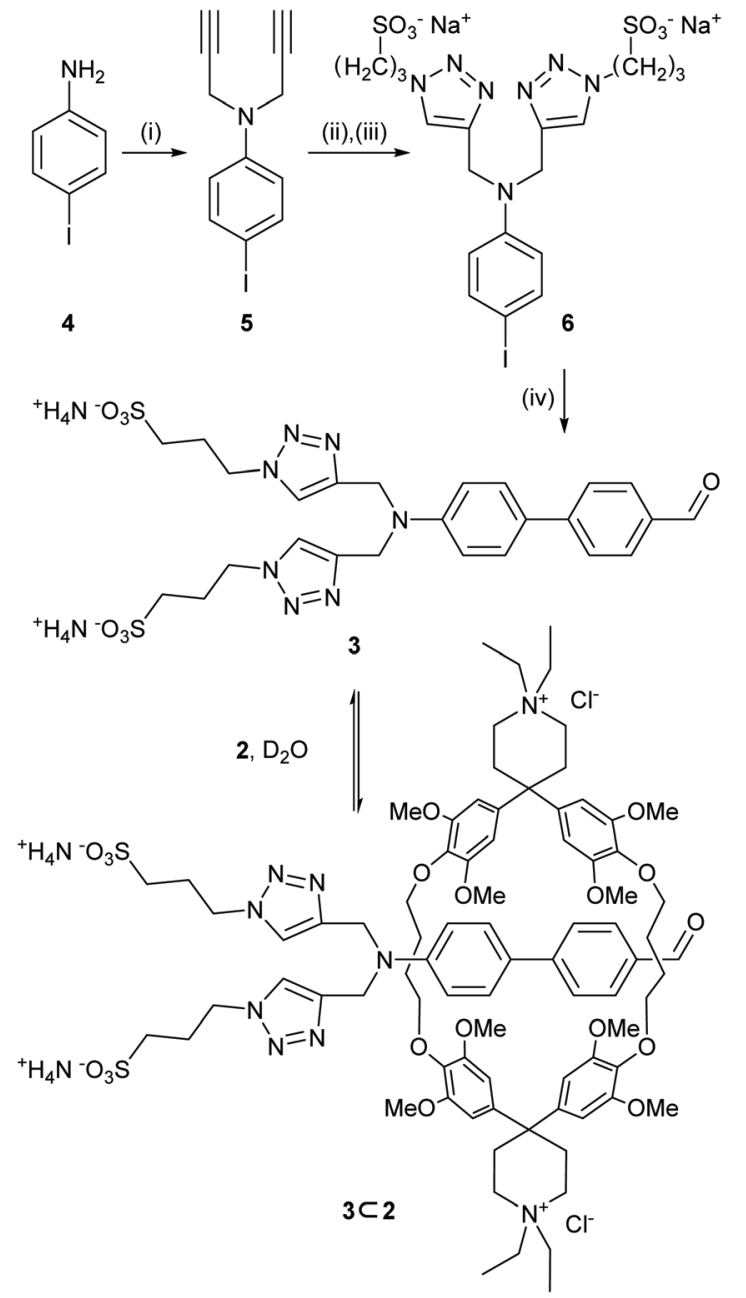

Scheme 2 Synthesis of 3 and pseudorotaxane formation with 2 in $\mathrm{D}_{2} \mathrm{O}$. (i) Propargyl bromide (30 wt $\%$ in toluene), $\mathrm{K}_{2} \mathrm{CO}_{3}, \mathrm{ACN}, 60{ }^{\circ} \mathrm{C}, 3 \mathrm{~d}, 66 \%$; (ii) $\mathrm{NaN}_{3}, 1,3$-propanesultone, $\mathrm{DMF} / \mathrm{H}_{2} \mathrm{O}(2: 1), \mathrm{rt}, 1 \mathrm{~h}$; (iii) TBTA, Cu $\left(\mathrm{CH}_{3} \mathrm{CN}\right)_{4} \mathrm{PF}_{6}$, sodium ascorbate, 5, rt, $22 \mathrm{~h}, 45 \%$; (iv) 4-formylphenylboronic acid, $\mathrm{K}_{3} \mathrm{PO}_{4}, \mathrm{Pd}(\mathrm{OAc})_{2}, \mathrm{H}_{2} \mathrm{O}$, reflux, $1 \mathrm{~h}, 78 \%$.

\section{Aqueous Knoevenagel condensations}

Having confirmed the formation of $3 \subset 2$ by ${ }^{1} \mathrm{H}-\mathrm{NMR}$ spectroscopy, we investigated conditions for the Knoevenagel condensation in aqueous media (Scheme 3). Inspired by literature procedures, ${ }^{49,50}$ we tested the condensation of malononitrile with 3 in water at room temperature in absence of any catalyst and were delighted to obtain 1a in 69\% yield after simple precipitation of the product by addition of acetonitrile. The "push-pull" dye 1a showed interesting optical properties (Fig. S14†) with an absorption maximum of $\lambda_{\mathrm{abs}}=420 \mathrm{~nm}$ and emission extending into the NIR with a maximum of $\lambda_{\text {em }}=710 \mathrm{~nm}$ in $\mathrm{H}_{2} \mathrm{O}$. The large Stokes' shift of approx. $9700 \mathrm{~cm}^{-1}$ observed for 1a was particularly appealing for both, biological applications and the optimization of superstructures for single molecule experiments profiting from the field enhancement in gold nanostructures.

Motivated by both, the encouraging preliminary synthesis and the exciting optical properties of the Knoevenagel product 1a, a large variety of sterically more demanding precursors were synthesized. From a molecular design view point, it seemed that the required methylene subunit trapped between a pair of EWGs was ideally suited to be decorated with bulky substituents, providing the option to profit from the Knoevenagel condensation to introduce the stopper required for the mechanically interlocked rotaxane structure. The overview and evaluation of considered methylene precursors comprising in part the stopper function is summarized in Scheme 3. Diester b was synthesized in an acid-catalyzed esterification of malonic acid; barbituric acid derivative $\mathbf{c}$ was obtained in two steps from a literature known urea derivative $^{51}$ (Scheme S4†). Unfortunately, the diester $\mathbf{b}$ turned out to be unserviceable for the intended purpose in preliminary experiments. The compound was not reactive at room temperature and its ester moieties were prone to hydrolysis at elevated temperatures under all reaction conditions investigated (acidic, basic, and uncatalyzed aqueous conditions). At the first glance, preliminary experiments with the barbituric acid derivative c were more promising, as the colour of the reaction mixture changed immediately to red upon addition of acetic acid to an aqueous solution of the reactants, pointing at the successful product formation. However, reaction control by LC-MS and reverse-phase TLC merely showed the starting materials. Repeating the experiment in $\mathrm{D}_{2} \mathrm{O}$, addition of $\mathrm{CD}_{3} \mathrm{CO}_{2} \mathrm{D}$ provided the expected red colour, yet it was not accompanied by any change in the ${ }^{1} \mathrm{H}-\mathrm{NMR}$ spectrum, likely due to the formation of only negligible amount of the intensely coloured desired product. Unfortunately, reaction conditions pushing the equilibrium towards the formation of 1c could not be found and we suspected the product to be of limited stability in aqueous medium.

We thus considered the less electron-withdrawing but likely more stable, commercially available 4,4'-methylenedibenzoic acid $\mathbf{d}$ next. Its reactivity was very poor even at elevated temperatures and reactions under more classic Knoevenagel conditions (DMF/piperidine) did not proceed either.

Puzzled by the refusal of the so far investigated methylene precursors to form the Knoevenagel product in aqueous media, we wondered whether their bulkiness or their charged state compromised either their reactivity or the stability of their condensation products. In a last attempt we developed the less symmetric precursor e, which features a bulky stopper on only one side of the active methylene component. In this compound the conjugation between the active methylene group and the stopper is broken by the methylene bridge between the amide and the triazole, thus the charged stopper is not participating in the electron withdrawal, unlike in molecules b-d. Of course we hoped that this variation in the molecular design will improve the reactivity of $\mathbf{e}$ and the stability of the resulting product $\mathbf{1 e}$.

The synthesis of e was performed in three steps from the literature-reported $^{52}$ aniline 7 (Scheme 4). First, a diazotation-azidation procedure gave 8 in $98 \%$ yield, followed by basic ester hydrolysis in THF/MeOH/ $\mathrm{H}_{2} \mathrm{O}$ to produce the stopper 9 in $96 \%$ yield after centrifugation and removal of excess $\mathrm{NaOH}$. In a final step, CuAAC with $\mathbf{f}$ led to the formation of $\mathbf{e}$ in $65 \%$ yield. 

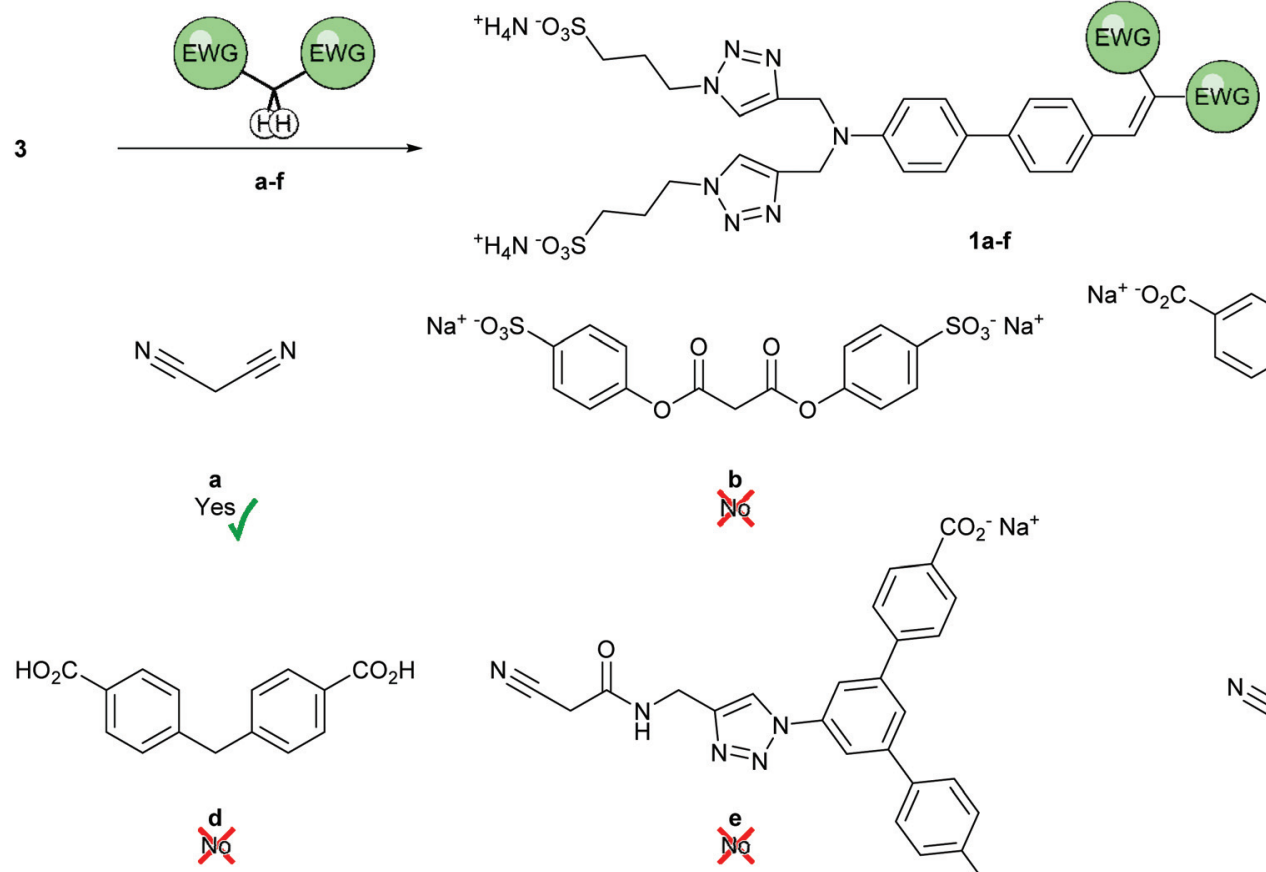<smiles>O=C(CC(=O)Oc1ccc(OS(=O)(=O)[O-])cc1)Oc1ccc(O[Na])cc1</smiles><smiles>[NH3+]OC(=O)c1ccc(N2C(=O)CC(=O)N(c3ccc(C(=O)[OH2+])cc3)C2=O)cc1</smiles><smiles>C[13C](C)(C)O[Na]</smiles>

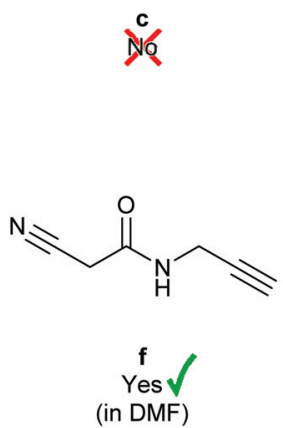

Scheme 3 Series of methylene precursors a-f activated by electron-withdrawing groups (EWGs) for the Knoevenagel reaction with 3 with the intention to form 1a-f. Answer statements together with ticks and crosses show the success in the search for suitable conditions for the formation of the corresponding Knoevenagel products in isolable yields.

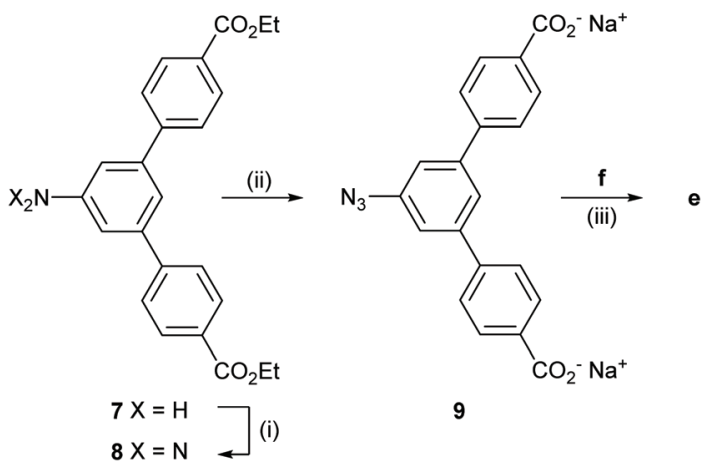

Scheme 4 Synthesis of the stopper 9 in 2 steps and CuAAC "click" reaction providing e. Reagents and conditions: (i) $\mathrm{TMSN}_{3}, \mathrm{tBuONO}, \mathrm{DMF}, 0^{\circ} \mathrm{C}$ to rt, $6 \mathrm{~h}, 98 \%$; (ii) $\mathrm{NaOH}$, THF-MeOH- $\mathrm{H}_{2} \mathrm{O}(4: 4: 1), 50{ }^{\circ} \mathrm{C}, 1 \mathrm{~h}, 86 \%$; (iii) $\mathrm{CuSO}_{4} \cdot 5 \mathrm{H}_{2} \mathrm{O}$, sodium ascorbate, $\mathrm{H}_{2} \mathrm{O}-\mathrm{MeOH}(2: 1), \mathrm{rt}, 1 \mathrm{~h}, 65 \%$.

However, the Knoevenagel condensation of e and 3 to form 1e (Scheme 3) remained challenging in aqueous conditions. In absence of catalyst the reaction did not proceed towards the product even at elevated temperatures, while addition of $\mathrm{Na}_{2} \mathrm{CO}_{3}$ led to amide hydrolysis already at room temperature. By adjusting $\mathrm{pH}$ to $\sim 7-8$ with a phosphate buffer, decomposition at elevated temperature was slowed down, however, the formation of the desired condensation product 1e was not observed. Acidic reaction conditions turned out to be even more challenging as the solubility of e decreased, substantially handicapping the formation of condensation product.
The reaction sequence forming the methylene precursor $\mathbf{e}$ with stopper potential by CuAAC "click" chemistry first and its subsequent engagement in the Knoevenagel condensation could also be inverted. The condensation could also be performed with the alkyne-bearing $\mathbf{f}$ and the obtained product exposed to the CuAAC "click" conditions to be further decorated by the bulky stopper. And indeed, the desired condensation product 1f was obtained, even so not in aqueous conditions. However, 1f profits from rather compact olefin substituents introduced by the Knoevenagel reaction, which should allow the subsequent rotaxane formation. As aqueous conditions are only required to unleash hydrophobic interaction to trigger the pseudorotaxane formation, the use of alternative solvents to obtain $\mathbf{1 f}$ is less an issue than in the case of bulkier methylene precursors. The formation of 1 f was observed in DMF with piperidine as catalyst. However, under these conditions $\mathbf{1 f}$ was prone to react further in a Michael addition. To our delight, omission of piperidine led to the $E$-selective (as evidenced by the NOESY NMR spectrum, Fig. S13†) formation of $\mathbf{1 f}$ in $36 \%$ isolated yield.

The absorption and emission spectra of 1 if in $\mathrm{H}_{2} \mathrm{O}$ (Fig. $\mathrm{S} 15 \dagger$ ) show a hypsochromic shift with respect to $\mathbf{1 a}$, with maxima of $396 \mathrm{~nm}$ and $693 \mathrm{~nm}$ for the absorption and emission of 1f, respectively. The Stokes' shift is, with a value of approximately $10800 \mathrm{~cm}^{-1}$, even larger than the one observed for $1 \mathrm{a}$.

As already mentioned above, due to the relatively compact $\mathbf{f}$, we anticipated that 2 should readily thread onto 1f. And 
indeed, the ${ }^{1} \mathrm{H}$-NMR analysis of a $1 \mathrm{mM} 1: 1$ mixture in $\mathrm{D}_{2} \mathrm{O}$ displayed the expected shifts of the aromatic signals of $\mathbf{1 f}$ (Scheme $\mathrm{S} 2 \dagger$ ). In similarity to $3 \subset 2$, the central protons of the biphenyl system in $\mathbf{1 f} \subset 2$ experienced the largest shift, suggesting the centre of the biphenyl subunit to be the most prevalent position of the host 2 in the superstructure. This is further corroborated by NOE signals of these central protons to the aromatic signal of 2 (Fig. S4 $\dagger$ ). ${ }^{1} \mathrm{H}-\mathrm{NMR}$ spectra of a dilution series (Fig. S5†) showed no significant changes in the chemical shift down to concentrations of $50 \mu \mathrm{M}$, indicating a $K_{\mathrm{a}}$ beyond the range suited for determination by NMR spectroscopy $\left(>10^{5} \mathrm{M}^{-1}\right)$. The association of $\mathbf{1 f} \subset 2$ in water was determined by recording the emission intensity during the titration of $\mathbf{1 f}$ against 2 (Fig. S11 $\dagger$ ). The obtained $K_{\mathrm{a}}$ of $1.5 \times$ $10^{7} \mathrm{M}^{-1}$ is larger than previously reported association constants with the same cyclophane, ${ }^{42,46}$ which might be the result of supporting electrostatic attractions between the negatively charged sulfonate groups of the axis and the piperidinium moieties of the macrocycle.

With the successful syntheses of e and 1f, together with its strong association with 2, our focus moved away from the original idea of using a Knoevenagel condensation as stoppering reaction (strategy A in Scheme 1a) to the stepwise assembly strategy (strategy B in Scheme 1a). Finally accepting that aqueous conditions are a challenging environment to push a reversible condensation reaction to completion, we wondered if a rotaxane with an axle assembled by a Knoevenagel condensation might be stable in water at all. Furthermore, we hoped to access the model compound to be able to assess the influence of the integration of the $\mathrm{D}-\pi-\mathrm{A}$ dye systems into the rotaxane superstructure on its optical properties.

\section{[2]Rotaxane}

With the confirmed formation of the pseudorotaxane $\mathbf{1 f} \subset \mathbf{2}$, the stoppering with 9 via CuAAC "click" reaction was envisioned (Scheme 5).
To simplify purification, classical conditions with $\mathrm{CuSO}_{4}$ and sodium ascorbate in absence of a ligand were chosen. The reaction was performed at $2 \mathrm{mM}$ concentration of 2 in $\mathrm{H}_{2} \mathrm{O}$, to prevent its self-aggregation. ${ }^{39}$ Higher host concentrations led to the formation of precipitate during the reaction. After $30 \mathrm{~min}$, we were delighted to observe the formation of new species with masses of the desired [2] rotaxane 1ec2 and the unthreaded side product 1e by LC-MS. While the ratio of both products could not be determined, we proceeded with the challenging isolation of the [2] rotaxane.

The solubility of $1 \mathrm{ec} 2$ was lower in $\mathrm{H}_{2} \mathrm{O}$ than that of its precursors, however it turned out to be well soluble in mixed solvents of $\mathrm{H}_{2} \mathrm{O} / \mathrm{CH}_{3} \mathrm{CN}$ and $\mathrm{H}_{2} \mathrm{O} / \mathrm{DMF}$, as well as in DMSO. While reverse-phase HPLC on C18-functionalized silica using $\mathrm{H}_{2} \mathrm{O} / \mathrm{CH}_{3} \mathrm{CN}$ as eluent allowed to remove most impurities like 1e, 2, and 9 over multiple consecutive runs, some remaining impurities could also not be removed using $\mathrm{H}_{2} \mathrm{O} / \mathrm{MeOH}$ eluent mixtures. Numerous alternative purification attempts like precipitations and crystallizations by vapour diffusion failed. Dialysis against water showed no significant improvement by ${ }^{1} \mathrm{H}$-NMR spectroscopy. Purification by gel permeation chromatography (GPC) using polystyrene beads (BioBeads SX-1) was considered, but the low solubility of $1 \mathrm{e} \subset 2$ in pure DMF and the poor swelling behaviour of the beads in polar protic media rendered the method ill-suited for this purpose. Finally, GPC using Sephadex LH-20 in a mixture of DMF and aqueous $\mathrm{NH}_{4} \mathrm{OAc}$ buffer, followed by a second dialysis, provided the rotaxane $1 \mathrm{ec} 2$ in $0.9 \%$ isolated yield with only minor impurities remaining. 1ec2 was characterized by ${ }^{1} \mathrm{H}-\mathrm{NMR}$ and 2D-NMR in $\mathrm{D}_{2} \mathrm{O} / \mathrm{CD}_{3} \mathrm{CN}$, and high-resolution mass spectrometry. The sparse availability of the mechanically interlinked model compound did neither allow to record ${ }^{13} \mathrm{C}-\mathrm{NMR}$ spectra showing all expected signals nor its characterization by elemental analysis, however, the chemical shifts of the missing ${ }^{13} \mathrm{C}$ peaks were extracted from HMQC and HMBC measurements.

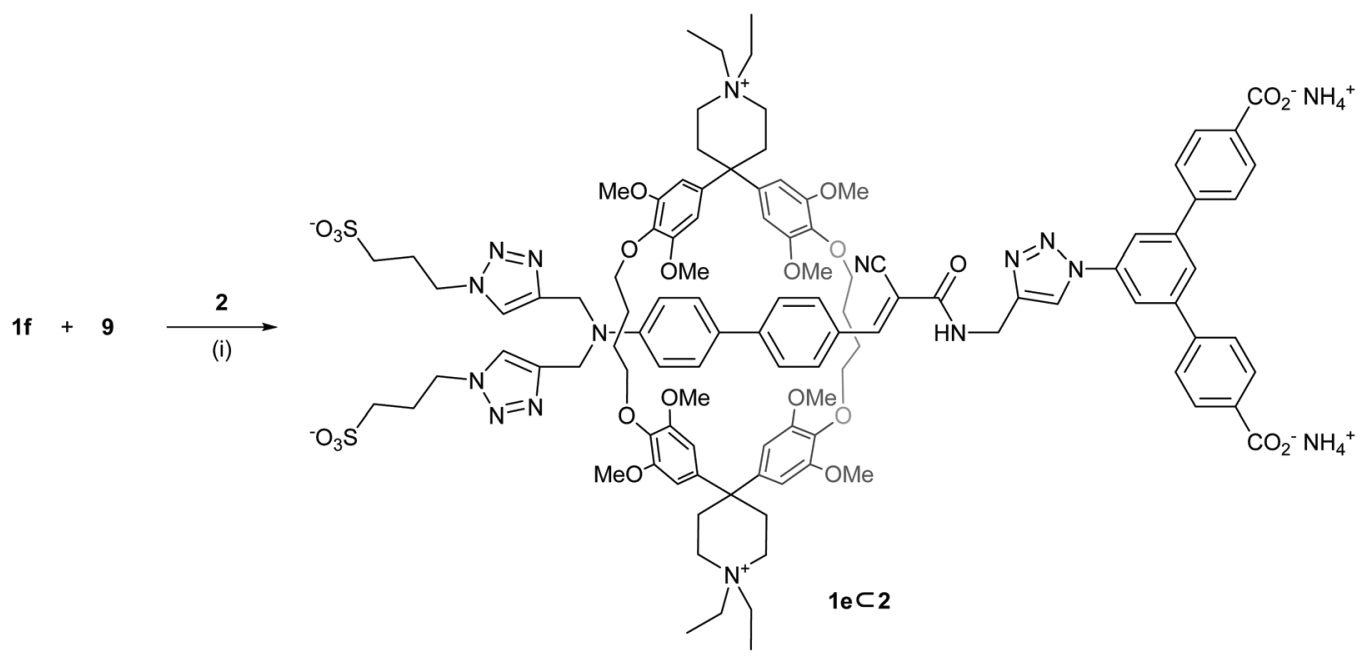

Scheme 5 Mechanical fixation in the rotaxane $1 \mathrm{ec} 2$ via CuAAC "click" reaction of $1 \mathrm{f}$ and 9 in presence of host 2 in aqueous environment. Reagents and conditions: (i) $\mathrm{CuSO}_{4} \cdot 5 \mathrm{H}_{2} \mathrm{O}$, sodium ascorbate, $\mathrm{H}_{2} \mathrm{O}, \mathrm{rt}, 1.5 \mathrm{~h}, 0.9 \%$. 
The low yield of the mechanically interlocked product led us to investigate the reaction further and ${ }^{1} \mathrm{H}-\mathrm{NMR}$ studies revealed the formation of undesired pseudorotaxane $\mathbf{9} \subset \mathbf{2}$ when 9 and 2 were mixed in $\mathrm{D}_{2} \mathrm{O}$ (Fig. S6†). Furthermore, equimolar competition experiments of 1f, 9, and 2 in $\mathrm{D}_{2} \mathrm{O}$ (Fig. S9†) revealed the partial formation of both $1 \mathrm{ec} 2$ and 9с2, pointing towards similarly high $K_{\mathrm{a}}$ values. Unfortunately, higher ratios of 2 led to precipitation during the CuAAC reaction and formation of impurities which were inseparable by HPLC, GPC, or precipitation.

To exclude that deslipping of the macrocycle off the axle in the [2] rotaxane occurred during purification, we heated solutions of $1 \mathrm{e} \subset 2$ in acetonitrile and DMSO to $75^{\circ} \mathrm{C}$ and $120{ }^{\circ} \mathrm{C}$, respectively. Qualitative analysis by HPLC-MS (Fig. S27†) showed neither the formation of 1e nor 2 after 16 h of heating, confirming sufficient bulkiness of both stopper moieties.

The unthreaded reference compound 1e was prepared by CuAAC between 1f and 9, and obtained in $81 \%$ yield after precipitation from the reaction mixture with an excess of $\mathrm{CH}_{3} \mathrm{CN}$ (Scheme S6†). Interestingly, 1e turned out to be better soluble in water than the [2] rotaxane. However, ${ }^{1} \mathrm{H}-\mathrm{NMR}$ spectra of $\mathbf{1 e}$ displayed broad signals in both, $\mathrm{D}_{2} \mathrm{O}$ and $\mathrm{D}_{2} \mathrm{O} / \mathrm{CD}_{3} \mathrm{CN}$ solutions, presumably due to the presence of different protonation states. Reasonably sharp NMR spectra were obtained in DMSO after addition of acetic acid.

To exclude solvent effects, the ${ }^{1} \mathrm{H}$-NMR spectra of $1 \mathrm{e}$ and 1 ec2 were compared in DMSO- $d_{6}$ with acetic acid as additive (Fig. 1). Akin to the NMR spectra of pseudorotaxanes $3 \subset 2$ and 1f $\subset 2$ measured in $\mathrm{D}_{2} \mathrm{O}$, the aromatic protons of the biphenyl moiety are most affected by the cyclophane, showing a significant upfield shift. The protons of the stoppers remain largely unaffected, further corroborating the biphenyl bridge as the preferred resting position of the cyclophane in the superstructure.

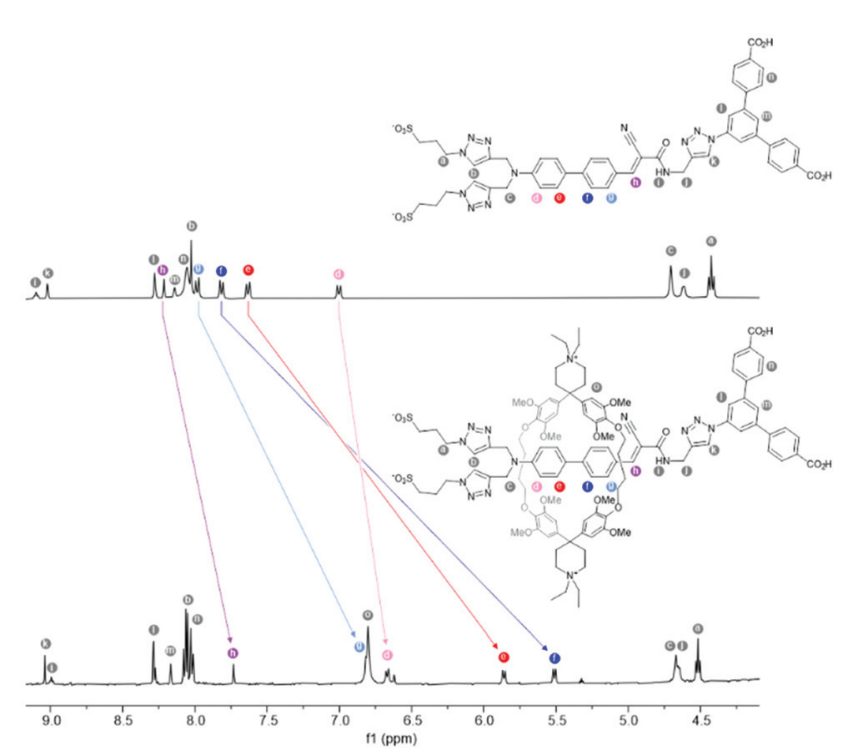

Fig. 1 Comparison of ${ }^{1} \mathrm{H}-\mathrm{NMR}$ spectra of $1 \mathrm{e}$ (top, $400 \mathrm{MHz}$ ) and $1 \mathrm{ec} 2$ (bottom, $500 \mathrm{MHz}$ ) in DMSO- $d_{6}$ with $\mathrm{AcOH}$ as additive.
We are mainly interested in the effects the cyclophane exercises on the optical properties of the $\mathrm{D}-\pi-\mathrm{A}$ chromophore, with the intention to develop model compounds for experiments investigating single molecule motions spectroscopically. Thus the optical properties of $1 \mathbf{e}$ and $\mathbf{1 e} \subset 2$ (Fig. 2a) were recorded in aqueous solutions containing $0.1 \mathrm{M} \mathrm{Na} \mathrm{NPO}_{4}$ adjusted to $\mathrm{pH} 6.4$ with $\mathrm{H}_{3} \mathrm{PO}_{4}$ to guarantee similar ions and comparable protonation states. In the UV-Vis absorption spectra, the longest wavelength absorption maxima are $420 \mathrm{~nm}$ and $406 \mathrm{~nm}$ for $1 \mathrm{e} \subset 2$ and 1e, respectively, showing a bathochromic shift in the [2] rotaxane. Exciting both samples at their absorption maxima for the emission spectra, displayed a hypsochromically shifted emission for $\mathbf{1 e c 2}$ compared to $\mathbf{1 e}$ with maxima at 655 and $669 \mathrm{~nm}$, respectively. More importantly, when corrected for the different absorptions, we noticed that the signal intensity at the emission maximum is significantly higher in the [2]rotaxane than in the unthreaded chromophore in the buffered aqueous solution. Intrigued by this result, we determined the absolute quantum yields (QY) in buffered aqueous solution to be below 1\% for 1e, while an efficiency of $11.0 \%( \pm 0.5 \%)$ was observed for $1 \mathrm{e} \subset 2$ under the same conditions (see ESI $\dagger$ ). In DMSO, the effect seemed to persist (Fig. S16†) and indeed, both molecules showed higher
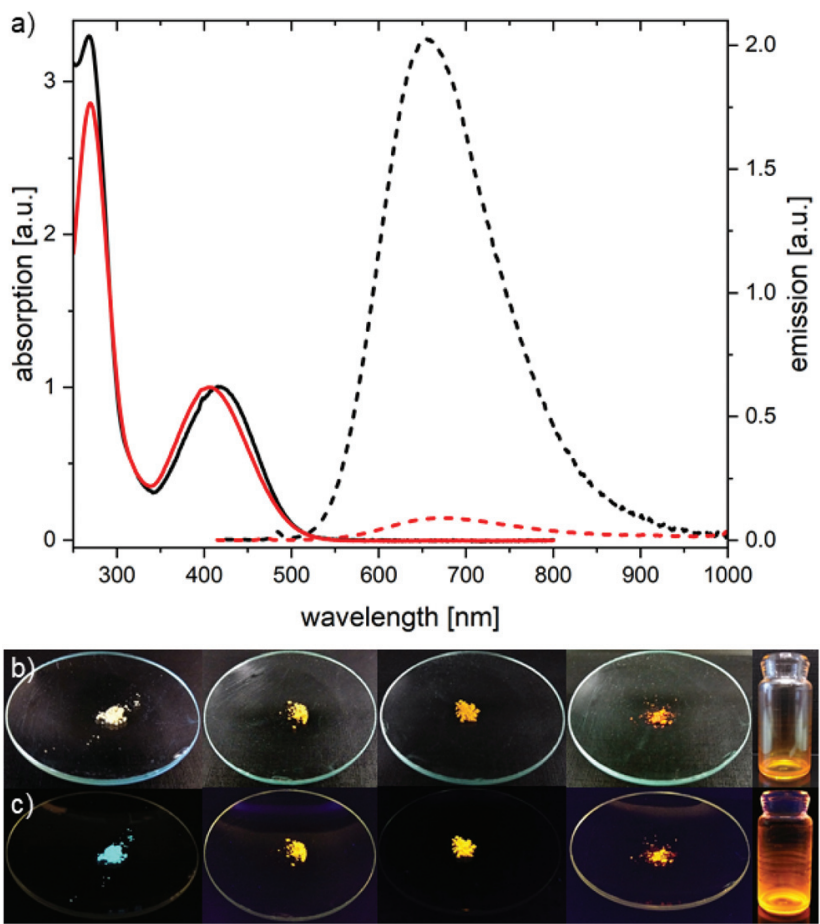

Fig. 2 (a) Absorption (solid lines) and emission (dashed lines) spectra of 1ec2 (black) and 1e (red) in aqueous $0.1 \mathrm{M} \mathrm{Na}_{2} \mathrm{HPO}_{4}$ solution adjusted to pH 6.4 with $\mathrm{H}_{3} \mathrm{PO}_{4}$. Absorption spectra are normalized. Emission spectra were corrected for differences in absorption at the excitation wavelength (420 nm for 1ec2; $406 \mathrm{~nm}$ for 1e). Photographs of 3, 1a, 1f, 1e, 1 ec2 (left to right) under ambient (b) and $366 \mathrm{~nm}$ wavelength light (c). Photographs in (c) were slightly edited to decrease the blue discoloration caused by the lamp, unedited photographs can be found in the supporting information (Fig. S22-S26†). 
QY in DMSO with values of $19.0 \%( \pm 0.9 \%)$ and $58.5 \%( \pm 1.5 \%)$ for the rod and [2] rotaxane, respectively. The mechanisms determining the extent of emission enhancement upon threading are not fully clarified yet and various factors might contribute like e.g., reduced solvent interaction or decreased rotational freedom. Aggregation-induced emission quenching of 1e seems unlikely as potential mechanism, as all the synthesised chromophores $\mathbf{3}, \mathbf{1 a}, \mathbf{1 e}, \mathbf{1 f}$, and $\mathbf{1 e c 2}$ even emit in the solid state when irradiated with light of $366 \mathrm{~nm}$ (Fig. 2b and c). The emission spectra of the finely ground solids of 3 , $\mathbf{1 a}, \mathbf{1 e}$, and $\mathbf{1 f}$ in between two glass slides were recorded in an integrating sphere, while $\mathbf{1 e \subset 2}$ was measured as a film on glass due to the lack of material. Interestingly, the emission spectra of dyes 1a, 1e, 1f, and 1eC2 (Fig. S21 †) show no significant differences, sharing an emission maximum at roughly $600 \mathrm{~nm}$. This hypsochromic shift can be explained by the absence of solvent interaction.

\section{Knoevenagel reversibility}

During experiments examining the pH-dependence of $\mathbf{1 e}$ in NMR spectroscopy, we observed the instability of $1 \mathrm{e}$ in $\mathrm{D}_{2} \mathrm{O} /$ $\mathrm{CD}_{3} \mathrm{CN}(1: 1)$ in the presence of $\mathrm{Na}_{2} \mathrm{CO}_{3}$, as expected based on our previous results with $\mathbf{e}$, which showed decomposition in Knoevenagel condensation attempts performed under basic conditions. Over time, an aldehyde signal arose, along with the characteristic peaks of the biphenyl moiety present in our chromophore design. To our delight, the ${ }^{1} \mathrm{H}$-NMR spectra of the formed species and 3 (in $\mathrm{D}_{2} \mathrm{O} / \mathrm{CD}_{3} \mathrm{CN}, 1: 1$, Scheme $\mathrm{S} 3 \uparrow$ ) share identical chemical shifts. Further examination by LC-MS corroborated 3 as the main species formed after $20 \mathrm{~h}$ (Fig. S28†). While e decomposed under these conditions, the experiment nonetheless indicated the reversibility of the Knoevenagel condensation in water, a prerequisite for its employment as a stoppering reaction for the formation of rotaxanes under thermodynamic control.

\section{Conclusions}

The challenges we faced during our ongoing journey studying the potential of the Knoevenagel condensation as stoppering reaction in water for the formation of rotaxanes are reported. The rigid-rod type water-soluble aldehyde precursor readily associated with the macrocyclic host in water and was suited for the synthesis of push-pull red to NIR-emitting dyes with solid-state emission. While we have not yet succeeded in finding a suitable reaction system enabling stoppering via Knoevenagel condensation, we synthesized and characterized a first "proof-of-existence" [2]rotaxane with a Knoevenagel product as axle. Significant emission enhancement was observed for the encapsulated dye in comparison to the unthreaded dye when measured in buffered aqueous solution and solid-state emission was preserved.

We are currently working on mechanically interlocked model compounds for externally triggered single molecule experiments.

\section{Conflicts of interest}

There are no conflicts to declare.

\section{Acknowledgements}

Generous financial support by the Volkswagen-Stiftung (Az. 93438) is gratefully acknowledged. M. M. acknowledges support from the 111 project (Grant No. 90002-18011002).

\section{Notes and references}

1 X. Ma and H. Tian, Bright functional rotaxanes, Chem. Soc. Rev., 2010, 39, 70-80.

2 M. Xue, Y. Yang, X. Chi, X. Yan and F. Huang, Development of Pseudorotaxanes and Rotaxanes: From Synthesis to Stimuli-Responsive Motions to Applications, Chem. Rev., 2015, 115, 7398-7501.

3 P. Rajamalli, F. Rizzi, W. Li, M. A. Jinks, A. K. Gupta, B. A. Laidlaw, I. D. W. Samuel, T. J. Penfold, S. M. Goldup and E. Zysman-Colman, Using the Mechanical Bond to Tune the Performance of a Thermally Activated Delayed Fluorescence Emitter, Angew. Chem., Int. Ed., 2021, 60, 12066-12073.

4 J. E. M. Lewis, M. Galli and S. M. Goldup, Properties and emerging applications of mechanically interlocked ligands, Chem. Commun., 2017, 53, 298-312.

5 Z.-Q. Cao, H. Li, J. Yao, L. Zou, D.-H. Qu and H. Tian, A Perylene-Bridged Switchable [3]Rotaxane Molecular Shuttle with a Fluorescence Output, Asian J. Org. Chem., 2015, 4, 212-216.

6 B. J. Slater, E. S. Davies, S. P. Argent, H. Nowell, W. Lewis, A. J. Blake and N. R. Champness, A Perylene Diimide Rotaxane: Synthesis, Structure and Electrochemically Driven De-Threading, Chem. - Eur. J., 2011, 17, 14746-14751.

7 A. H. G. David, P. García-Cerezo, A. G. Campaña, F. Santoyo-González and V. Blanco, [2]Rotaxane EndCapping Synthesis by Click Michael-Type Addition to the Vinyl Sulfonyl Group, Chem. - Eur. J., 2019, 25, 6170-6179.

8 D. D. Günbaş, L. Zalewski and A. M. Brouwer, Solvatochromic rotaxane molecular shuttles, Chem. Commun., 2011, 47, 4977.

9 S. Anderson, T. D. W. Claridge and H. L. Anderson, Azo-Dye Rotaxanes, Angew. Chem., Int. Ed., 1997, 36, 1310-1313.

10 H. Yan, L. Zhu, X. Li, A. Kwok, X. Li, H. Ågren and Y. Zhao, Photothermal-responsive [2]rotaxanes, RSC Adv., 2013, 3, 2341.

11 M. R. Craig, T. D. W. Claridge, H. L. Anderson and M. G. Hutchings, Synthesis of a cyclodextrin azo dye [3] rotaxane as a single isomer, Chem. Commun., 1999, 15371538.

12 M. Xue, Y.-S. Su and C.-F. Chen, Isomeric Squaraine-Based [2]Pseudorotaxanes and [2]Rotaxanes: Synthesis, Optical Properties, and Their Tubular Structures in the Solid State, Chem. - Eur. J., 2010, 16, 8537-8544. 
13 J. J. Gassensmith, J. M. Baumes and B. D. Smith, Discovery and early development of squaraine rotaxanes, Chem. Commun., 2009, 6329-6338.

14 W. Zhou, J. Li, X. He, C. Li, J. Lv, Y. Li, S. Wang, H. Liu and D. Zhu, A Molecular Shuttle for Driving a Multilevel Fluorescence Switch, Chem. - Eur. J., 2008, 14, 754-763.

15 D. A. Leigh, M. Á. F. Morales, E. M. Pérez, J. K. Y. Wong, C. G. Saiz, A. M. Z. Slawin, A. J. Carmichael, D. M. Haddleton, A. M. Brouwer, W. J. Buma, G. W. H. Wurpel, S. León and F. Zerbetto, Patterning through Controlled Submolecular Motion: Rotaxane-Based Switches and Logic Gates that Function in Solution and Polymer Films, Angew. Chem., Int. Ed., 2005, 44, 3062-3067.

16 T. Ogoshi, D. Yamafuji, T. Yamagishi and A. M. Brouwer, Förster resonance energy transfer by formation of a mechanically interlocked [2]rotaxane, Chem. Commun., 2013, 49, 5468.

17 W. Zhou, J. Xu, H. Zheng, H. Liu, Y. Li and D. Zhu, Charge Transfer Chromophore-Stopped [2]Rotaxane through [2+2] Cycloaddition, J. Org. Chem., 2008, 73, 7702-7709.

18 H. Zhang, Q. Wang, M. Liu, X. Ma and H. Tian, Switchable V-Type [2]Pseudorotaxanes, Org. Lett., 2009, 11, 3234-3237.

19 S. J. Vella, J. Tiburcio, J. W. Gauld and S. J. Loeb, Push-Pull [2]Pseudorotaxanes. Electronic Control of Threading by Switching ON/OFF an Intramolecular Charge Transfer, Org. Lett., 2006, 8, 3421-3424.

20 L. S. Atabekyan, A. I. Vedernikov, V. G. Avakyan, N. A. Lobova, S. P. Gromov and A. K. Chibisov, Photoprocesses in styryl dyes and their pseudorotaxane complexes with cucurbit[7]uril, J. Photochem. Photobiol. A. Chem., 2013, 253, 52-61.

21 L. Zhu, X. Li, F.-Y. Ji, X. Ma, Q.-C. Wang and H. Tian, Photolockable Ratiometric Viscosity Sensitivity of Cyclodextrin Polypseudorotaxane with Light-Active Rotor Graft, Langmuir, 2009, 25, 3482-3486.

22 J. E. H. Buston, J. R. Young and H. L. Anderson, Rotaxaneencapsulated cyanine dyes: enhanced fluorescence efficiency and photostability, Chem. Commun., 2000, 905906.

23 J. E. H. Buston, H. L. Anderson and F. Marken, Enhanced chemical reversibility of redox processes in cyanine dye rotaxanes, Chem. Commun., 2001, 1046-1047.

24 F. Bureš, Fundamental aspects of property tuning in pushpull molecules, RSC Adv., 2014, 4, 58826-58851.

25 H. Lahlali, K. Jobe, M. Watkinson and S. M. Goldup, Macrocycle Size Matters: "Small” Functionalized Rotaxanes in Excellent Yield Using the CuAAC Active Template Approach, Angew. Chem., Int. Ed., 2011, 50, 4151-4155.

26 V. Aucagne, K. D. Hänni, D. A. Leigh, P. J. Lusby and D. B. Walker, Catalytic "Click" Rotaxanes: A Substoichiometric Metal-Template Pathway to Mechanically Interlocked Architectures, J. Am. Chem. Soc., 2006, 128, 2186-2187.

27 J. E. M. Lewis, P. D. Beer, S. J. Loeb and S. M. Goldup, Metal ions in the synthesis of interlocked molecules and materials, Chem. Soc. Rev., 2017, 46, 2577-2591.
28 N. Hoyas Pérez and J. E. M. Lewis, Synthetic strategies towards mechanically interlocked oligomers and polymers, Org. Biomol. Chem., 2020, 18, 6757-6780.

29 S. J. Cantrill, S. J. Rowan and J. F. Stoddart, Rotaxane Formation under Thermodynamic Control, Org. Lett., 1999, 1, 1363-1366.

30 I. Neira, A. Blanco-Gómez, J. M. Quintela, C. Peinador and M. D. García, Adjusting the Dynamism of Covalent Imine Chemistry in the Aqueous Synthesis of Cucurbit[7]urilbased [2]Rotaxanes, Org. Lett., 2019, 21, 8976-8980.

31 Y. Suzaki and K. Osakada, Ferrocene-containing [2]- and [3] rotaxanes. Preparation via an end-capping cross-metathesis reaction and electrochemical properties, Dalton Trans., 2007, 2376.

32 E. Knoevenagel, Condensation von Malonsäure mit aromatischen Aldehyden durch Ammoniak und Amine, Berichte Dtsch. Chem. Ges., 1898, 31, 2596-2619.

33 R. Chen, J.-L. Shi, Y. Ma, G. Lin, X. Lang and C. Wang, Designed Synthesis of a 2D Porphyrin-Based sp2 Carbon-Conjugated Covalent Organic Framework for Heterogeneous Photocatalysis, Angew. Chem., Int. Ed., 2019, 58, 6430-6434.

34 E. Jin, M. Asada, Q. Xu, S. Dalapati, M. A. Addicoat, M. A. Brady, H. Xu, T. Nakamura, T. Heine, Q. Chen and D. Jiang, Two-dimensional sp2 carbon-conjugated covalent organic frameworks, Science, 2017, 357, 673676.

35 S. Lee, C.-H. Chen and A. H. Flood, A pentagonal cyanostar macrocycle with cyanostilbene $\mathrm{CH}$ donors binds anions and forms dialkylphosphate [3]rotaxanes, Nat. Chem., 2013, 5, 704-710.

36 M. van Oosten, T. Schäfer, J. A. C. Gazendam, K. Ohlsen, E. Tsompanidou, M. C. de Goffau, H. J. M. Harmsen, L. M. A. Crane, E. Lim, K. P. Francis, L. Cheung, M. Olive, V. Ntziachristos, J. M. van Dijl and G. M. van Dam, Realtime in vivo imaging of invasive- and biomaterial-associated bacterial infections using fluorescently labelled vancomycin, Nat. Commun., 2013, 4, 2584.

37 R. Bardhan, N. K. Grady, J. R. Cole, A. Joshi and N. J. Halas, Fluorescence Enhancement by $\mathrm{Au}$ Nanostructures: Nanoshells and Nanorods, ACS Nano, 2009, 3, 744-752.

38 A. P. Francisco, D. Botequim, D. M. F. Prazeres, V. V. Serra, S. M. B. Costa, C. A. T. Laia and P. M. R. Paulo, Extreme Enhancement of Single-Molecule Fluorescence from Porphyrins Induced by Gold Nanodimer Antennas, J. Phys. Chem. Lett., 2019, 10, 1542-1549.

39 S. Anderson, R. T. Aplin, T. D. W. Claridge, T. Goodson III, A. C. Maciel, G. Rumbles, J. F. Ryan and H. L. Anderson, An approach to insulated molecular wires: synthesis of watersoluble conjugated rotaxanes, J. Chem. Soc., Perkin Trans. 1, 1998, 2383-2398.

40 S. B. Ferguson, E. M. Seward, F. Diederich, E. M. Sanford, A. Chou, P. Inocencio-Szweda and C. B. Knobler, Strong enthalpically driven complexation of neutral benzene guests in aqueous solution, J. Org. Chem., 1988, 53, 5593-5595. 
41 F. Diederich, Complexation of Neutral Molecules by Cyclophane Hosts, Angew. Chem., Int. Ed. Engl., 1988, 27, 362-386.

42 P. N. Taylor, A. J. Hagan and H. L. Anderson, Hindered fluorescence quenching in an insulated molecular wire, Org. Biomol. Chem., 2003, 1, 3851.

43 S. Anderson and H. L. Anderson, Synthesis of a WaterSoluble Conjugated [3]Rotaxane, Angew. Chem., Int. Ed. Engl., 1996, 35, 1956-1959.

44 Y. Aeschi, S. Drayss-Orth, M. Valášek, F. Raps, D. Häussinger and M. Mayor, Assembly of [2]Rotaxanes in Water: Assembly of [2]Rotaxanes in Water, Eur. J. Org. Chem., 2017, 2017, 4091-4103.

45 Y. Aeschi, S. Drayss-Orth, M. Valášek, D. Häussinger and M. Mayor, Aqueous Assembly of Zwitterionic Daisy Chains, Chem. - Eur. J., 2019, 25, 285-295.

46 Y. Aeschi, L. Jucker, D. Häussinger and M. Mayor, Slow Formation of Pseudorotaxanes in Water: Slow Formation of Pseudorotaxanes in Water, Eur. J. Org. Chem., 2019, 2019, 3384-3390.

47 Z. Li, C. Gelbaum, W. L. Heaner, J. Fisk, A. Jaganathan, B. Holden, P. Pollet and C. L. Liotta, Palladium-Catalyzed
Suzuki Reactions in Water with No Added Ligand: Effects of Reaction Scale, Temperature, $\mathrm{pH}$ of Aqueous Phase, and Substrate Structure, Org. Process Res. Dev., 2016, 20, 14891499.

48 P. Thordarson, Determining association constants from titration experiments in supramolecular chemistry, Chem. Soc. Rev., 2011, 40, 1305-1323.

49 F. Areias, M. Costa, M. Castro, J. Brea, E. Gregori-Puigjané, M. F. Proença, J. Mestres and M. I. Loza, New chromene scaffolds for adenosine A2A receptors: Synthesis, pharmacology and structure-activity relationships, Eur. J. Med. Chem., 2012, 54, 303-310.

50 F. Bigi, M. L. Conforti, R. Maggi, A. Piccinno and G. Sartori, Clean synthesis in water: uncatalysed preparation of ylidenemalononitriles, Green Chem., 2000, 2, 101-103.

51 A. Azhdari Tehrani, L. Esrafili, S. Abedi, A. Morsali, L. Carlucci, D. M. Proserpio, J. Wang, P. C. Junk and T. Liu, Urea Metal-Organic Frameworks for Nitro-Substituted Compounds Sensing, Inorg. Chem., 2017, 56, 1446-1454.

52 P. Martinez-Bulit, B. H. Wilson and S. J. Loeb, One-pot synthesis of porphyrin-based [5]rotaxanes, Org. Biomol. Chem., 2020, 18, 4395-4400. 